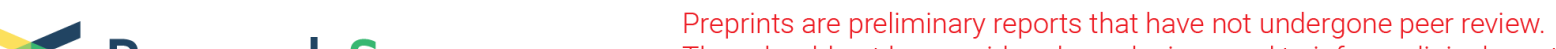 $\begin{array}{ll}\text { Research Square } & \begin{array}{l}\text { They should not be considered conclusive, used to inform clinical practice, } \\ \text { or referenced by the media as validated information. }\end{array}\end{array}$
}

\section{COVID-19 Vaccine Hesitancy Among Different Population Groups in China: a National Multi-Center Online Survey}

\section{Yiman Huang}

Chinese Academy of Medical Sciences \& Peking Union Medical College https://orcid.org/0000-00028236-1154

\section{Xiaoyou Su ( $\nabla$ suxiaoyou@hotmail.com )}

Chinese Academy of Medical Sciences \& Peking Union Medical College https://orcid.org/0000-00024216-2142

\section{Weijun Xiao}

Chinese Academy of Medical Sciences \& Peking Union Medical College

\section{Hao Wang}

Chinese Academy of Medical Sciences \& Peking Union Medical College

\section{Mingyu Si}

Chinese Academy of Medical Sciences \& Peking Union Medical College

\section{Wenjun Wang}

Jining Medical College: Jining Medical University

\section{Xiaofen Gu}

Xinjiang Medical University Affiliated Tumor Hospital

\section{Li Ma}

Dalian Medical College: Dalian Medical University

\section{Li Li}

Jinan University

\section{Shaokai Zhang}

Henan Provincial Tumor Hospital: Henan Cancer Hospital

\section{Chunxia Yang}

Sichuan University

\section{Yanqin Yu}

Baotou Medical College

\section{Youlin Qiao}

Chinese Academy of Medical Sciences \& Peking Union Medical College

\section{Research Article}


Keywords: vaccine hesitancy, COVID-19, public health professionals, infodemic, China

Posted Date: August 10th, 2021

DOI: https://doi.org/10.21203/rs.3.rs-757495/v1

License: (c) (1) This work is licensed under a Creative Commons Attribution 4.0 International License. Read Full License 


\section{Abstract}

Background: COVID-19 vaccine has been available in China since the beginning of the 2021, however, certain numbers of people are reluctant for some reasons to vaccinate. The high vaccine coverage is crucial for controlling disease transmission. Meanwhile, the vaccine hesitancy might be a barrier to the establishment of sufficient herd immunization. This study aims to investigate the prevalence of vaccine hesitancy towards the COVID-19 vaccine among different population groups, and explores the characteristics of different groups about vaccine hesitancy and common barriers and facilitators to vaccination decisions.

Methods: The current survey was performed among students, public health professionals, medical workers and general population from January to March 2021 among Chinese from seven cities located in seven geographical territories of China. The questionnaire contained sociodemographic information, concerns about COVID-19 epidemic, general vaccination behavior and attitudes, the General Vaccine Hesitancy Scale, the COVID-19 Vaccine Hesitancy Scale and other potential factors of vaccine hesitancy. Univariate analysis was conducted by chi-squared test, and variables were significant at $P<0.10$ were then included in a multivariable regression model.

Results: The prevalence of COVID-19 vaccine hesitancy was $64.1 \%$ in our study, and a higher prevalence $(73.7 \%)$ was in public health professionals compared to students $(58.3 \%)$, medical workers $(64.9 \%)$ and general population (61.1\%). The results of multivariate analysis indicated that public health professionals were more likely to be hesitant against COVID-19 vaccine than general population (OR: $1.469,95 \% \mathrm{Cl}$ : 1.069-2.019. And participants who needed transparent information about COVID-19 vaccine development, efficacy and safety (OR: $1.609,95 \% \mathrm{Cl}: 1.343-1.928)$ and who have received negative information of COVID-19 vaccine (OR: $1.300,95 \% \mathrm{Cl}: 1.130-1.496)$ were more likely to have vaccine hesitancy.

Conclusions: Appropriate training in knowledge and communication skills about vaccines are necessary for public health professionals to help themselves and the public to increase their willingness of vaccination. Reducing the spread of misinformation and disseminating facts in a timely and accurate way will likely reduce vaccine hesitancy. Also, to establish suitable communication strategies between the government and the public and a warning system on infodemic would be helpful to improve public's confidence in vaccination.

\section{Background}

COVID-19 (coronavirus disease 2019) was first noticed in late 2019 and soon caused a pandemic over the world(1). There is no doubt that COVID-19 has been an unprecedented crisis for global public health and is expected to continue to cause an enormous burden of morbidity and mortality while severely disrupting societies and economies. The spread of COVID-19 as a pandemic disease in the world requires immediate action to prevent the increase of cases and curb its worldwide spread, including the production of vaccines, which can be an effective measure to protect people against this disease $(2,3)$. 
Thus, China and some other countries have been devoted to develop safe and effective vaccines, and several vaccines are available at present. Although the COVID-19 vaccine is free for all citizens in China, it does not guarantee sufficient herd immunization due to widespread hesitancy. Therefore, compared to the availability of the vaccine, getting the COVID-19 vaccination is more important.

Vaccination was recognized as one of the most successful public health measures, and vaccination programs have contributed to the decline in mortality and morbidity of various infectious diseases (4). However, general public still have significant doubts and concerns regarding vaccination due to the multiple historical, political, socio-cultural context and personal factors (5). This phenomenon is described as vaccine hesitancy. Vaccine hesitancy refers to delay in acceptance or refusal of vaccination despite availability of vaccination services. Vaccine hesitancy is complex and context specific, varying across time, place and vaccines (6). Vaccination can prevent lots of diseases, especially infectious diseases, hence vaccine hesitancy can result in apparently increases in outbreaks, morbidity and mortality of some vaccine-preventable diseases. Indeed, the concept of "vaccine hesitancy" has been considered by the World Health Organization (WHO) as "one of the top-ten threats to global health" (7).

Various countries started rolling out vaccines in late 2020, prioritizing healthcare and other front-line workers and those with high-risk health conditions. But when the vaccine become fully available, certain amount of individuals are still unsure whether they should get the COVID-19 vaccine or not. According to the results of a previous study, respondents from China gave the highest proportion (88.6\%) of positive responses when asked if they would take a "proven, safe and effective vaccine" (8). Among healthcare workers in China, $76.63 \%$ declared they would accept the vaccine when it becomes available (9). Approximately $68 \%$ of all respondents were supportive of being vaccinated for COVID-19 in the United States, while side effects, efficacy and length of testing remained their major concerns (10-12). An online survey found that about one-third of the participants in Turkey and $14 \%$ in the UK were unsure about getting COVID-19 vaccine (13). Overall, there will always be people who are reluctant for some reasons to vaccinate, such as concerns over the short period of time of vaccine development and concerns about safety and efficacy. As we all known, the extraordinary herd protection is essential to the success of vaccination campaigns, but it can only be obtained with high vaccine coverage and the effectiveness of the COVID-19 vaccine in controlling disease transmission also depends on it (14).

However, the prevalence of COVID-19 vaccine hesitancy among different population groups in China was still unclear. It is also an urgent need to be investigated what factors may have influenced their hesitancy. To understand the prevalence of public's hesitancy on COVID-19 vaccines and related factors plays a principal role in implementing evidence-based interventions to increase the vaccination rate. This study investigates the prevalence of vaccine hesitancy towards the COVID-19 vaccine among different population groups from seven geographical territories of China, and explores the characteristics of different groups about vaccine hesitancy and common barriers and facilitators to vaccination decisions.

\section{Methods}




\section{1 study design and participants}

The current survey was performed from January to March 2021 among Chinese from seven cities (from Henan Province, Sichuan Province, Shandong Province, Guangdong Province, Inner Mongolia, Xinjiang Uygur Autonomous Region, and Liaoning Province respectively) located in seven geographical territories of China by distributing online questionnaire via an investigation platform named wenjuanxing. The participants were recruited from four different population groups, including students, public health professionals, medical workers and general population.

The eligibility criteria included age more than or equal to 18 and an ability to read, understand and complete an online questionnaire. Those who were younger than 18 , had barriers to use mobile phone or computer, or had cognitive impairment were excluded. To confirm the quality of the online survey, the members of research team were trained on data collection and inclusion criteria procedure.

\subsection{Measurements}

The survey questionnaire contained sociodemographic information, concerns about COVID-19 epidemic, general vaccination behavior and attitudes, the General Vaccine Hesitancy Scale, the COVID-19 Vaccine Hesitancy Scale and other potential factors of vaccine hesitancy.

\subsubsection{Sociodemographic information}

Sociodemographic variables included age, gender, ethnicity, residence place, marital status, education level, household income (during past 1 year), smoking and drinking history, and COVID-19 test results.

\subsubsection{Concerns of COVID - 19 pandemic}

We assessed the concerns of participants about COVID - 19 pandemic by a 3-items scale, which included "I am scared about getting infected with COVID-19," "The possibility of getting infected with COVID-19 in the future concerns me," and "I don't really worry about getting infected with COVID-19". This 3-item scale was developed and utilized from several studies, participants responded to each item on a 5-point Likerttype scale $(1=$ strongly disagree to $5=$ strongly agree) $(15,16)$. After reverse coded the last item, the three items were highly correlated with satisfactory reliability (Cronbach's $a=0.702$ ).

\subsubsection{The General Vaccine Hesitancy Scale}

The General Vaccine Hesitancy Scale composed of 10 items which was revised on the base of previous studies $(17,18)$. In this study, we used the 10 items of the Vaccine Hesitancy Scale (VHS) developed by the SAGE Working Group on Vaccine Hesitancy that are measured on a five-point Likert-type rating scale ranging from "strongly disagree" to "strongly agree". Corresponding changes were made to the wordings of the 10 items to make the study participants fully understand the meanings. We reversed seven items in the scoring of the scale so that higher scores indicated more hesitancy on all items. The reliability of the General Vaccine Hesitancy Scale was satisfactory in our study (Cronbach's $a=0.930$ ). 


\subsubsection{The COVID-19 Vaccine Hesitancy Scale}

The COVID-19 Vaccine Hesitancy Scale was measured by 15 items based on previous studies to identify vaccine-hesitant parents $(19,20)$. We collapsed responses of scale items into 3 categories: hesitant responses, "not sure or don't know", and non-hesitant responses. For the 6 items with a 5-point Likertscale ranging from "strongly agree to strongly disagree", hesitant responses were the answer of "strongly agree/agree" for 3 items and "strongly disagree/disagree" for 3 items. For 3 items with a 5-point Likertscale ranging from "not at all concerned to very concerned", hesitant response corresponded to the responses of "somewhat or very hesitant". For yes/no items, "yes" was considered to be the hesitant response and "no" the non-hesitant response. Lastly, for the items with an 11-point Likert-scale, hesitant responses corresponded to the collapsed responses $0-5$, not sure responses to $6-7$, and non-hesitant responses to $8-10$. Totally, the score was higher than or equal to 50 was indicated having COVID-19 vaccine hesitancy (20). The Cronbach's alpha of the 15-item scale in the current study was 0.719 .

\subsubsection{General vaccination behavior and attitudes}

The questionnaire used a series of questions to investigate respondents' vaccination history, including the following items: "Do you agree that vaccines can protect you from diseases?" "Do you agree that you will get all vaccines that National Immunization Program or government recommended?" "Have you ever hesitated to get vaccination?" "Have you ever refused to get vaccination?" "Have you ever hesitated or refused to be get Pneumococcal Vaccine?" "Have you ever hesitated or refused to get Influenza Vaccine?"

\subsubsection{Potential factors of COVID-19 vaccine hesitancy}

In this section, the following items were designed to explore the barriers of get COVID-19 vaccine and related factors of COVID-19 vaccine hesitancy: (1) individual attitudes to COVID-19: "Do you agree that COVID-19 epidemic is a severe problem affecting the health of the community?" "Do you agree that COVID-19 will be a great threat to your health if you are infected?" (2) significant people's advice: "Do you agree that you will get COVID-19 vaccine if doctors recommend?", "Do you agree that the advice of your family members or friends will affect your intention of getting COVID-19 vaccine?" (3) information about COVID-19 vaccine: "Do you need transparent information about COVID-19 vaccine development, efficacy and safety?" "Do you have doubts about the source of information about the COVID-19 vaccine?" "Have you ever received negative information about getting the COVID-19 vaccine?" "Would you like to get the COVID-19 vaccine after receiving the negative information about it?" (4) cost or time to get COVID-19 vaccine: "Do you agree that the time costs in waiting for the vaccination or staying at the clinic will be a barrier for you to get COVID-19 vaccine?" "Do you agree that the environment of the clinic will be a barrier for you to get COVID-19 vaccine?" "Do you agree that the cost of going to the clinic will be a barrier for you to get COVID-19 vaccine?" (5) personal conditions: "Do you agree that you have no need of getting COVID-19 vaccine because you are healthy?" "Have you gotten emergency COVID-19 vaccination for some reasons?".

\subsection{Statistical analysis}


This study described the sociodemographic information by counts and proportions among participants. The chi-squared test was utilized to compared the differences of various factors in different population groups. Survey responses were combined into two categories (having or not having COVID-19 vaccine hesitancy) according to whether the score of the COVID-19 Vaccine Hesitancy Scale was higher than or equal to 50. And ordinal regression models were ran to examine demographic and attitudinal factors predictive of respondents' hesitancy to get vaccinated against COVID-19. To identify suitable candidate variables for regression models, univariate analysis was first conducted by chi-squared test, and variables were significant at $\mathrm{P}<0.10$ were then included in a multivariable regression model. The level of significance was set at $P<0.05$. Data were analyzed by using SPSS version 24.0.

\section{Results}

\section{1 sociodemographic characteristics}

Table 1 summarized the characteristics distributions of participants by different population groups. A total of 4289 respondents (response rate $95.37 \%$ ) completed the online questionnaire, and 62 questionnaires were excluded due to the age limitations. Of them, there were 2656 (62.8\%) medical workers, $753(17.8 \%)$ students, $434(10.3 \%)$ general population, and $384(9.1 \%)$ public health professionals. The mean age was 33.02 years. In total, $2818(66.7 \%)$ respondents were female, $89.1 \%$ of them were Han ethnicity, the majority of participants (85.6\%) lived in urban areas and $56.9 \%$ of them were married, $41.3 \%$ were single. The education level of most of them $(93.8 \%)$ were college and above. For the household income during the past year, nearly half of them were in 50000-100000 Yuan per year. Most of participants self-reported they have not ever smoking (88.3\%) or drinking (84.6\%). 
Table 1

Sociodemographic characteristics of participants by different population groups

\begin{tabular}{|c|c|c|c|c|c|}
\hline Variables & $\begin{array}{l}\text { Medical } \\
\text { workers }(n= \\
2656)\end{array}$ & $\begin{array}{l}\text { Students(n } \\
=753)\end{array}$ & $\begin{array}{l}\text { General } \\
\text { population }(n= \\
\text { 434) }\end{array}$ & $\begin{array}{l}\text { Public health } \\
\text { professionals }(n= \\
384)\end{array}$ & $\begin{array}{l}\text { Total }(\mathrm{n} \\
=4227)\end{array}$ \\
\hline $\begin{array}{l}\text { Age (years) } \\
\text { (mean } \pm \text { SD) }\end{array}$ & $35.89 \pm 9.33$ & $\begin{array}{l}22.47 \pm \\
3.13\end{array}$ & $29.73 \pm 7.78$ & $37.52 \pm 9.03$ & $\begin{array}{l}33.02 \pm \\
9.90\end{array}$ \\
\hline \multicolumn{6}{|l|}{$\begin{array}{l}\text { Gender, } \mathrm{n} \\
\text { (\%) }\end{array}$} \\
\hline Male & $732(27.6)$ & $334(44.4)$ & $193(44.5)$ & $150(39.1)$ & $\begin{array}{l}1409 \\
(33.3)\end{array}$ \\
\hline Female & $1924(72.4)$ & $419(55.6)$ & $241(55.5)$ & $234(60.9)$ & $\begin{array}{l}2818 \\
(66.7)\end{array}$ \\
\hline \multicolumn{6}{|l|}{$\begin{array}{l}\text { Ethnicity, n } \\
\text { (\%) }\end{array}$} \\
\hline Han & $2339(88.1)$ & $654(86.9)$ & $415(95.6)$ & $357(93.0)$ & $\begin{array}{l}3765 \\
(89.1)\end{array}$ \\
\hline Other & 317 (11.9) & $99(13.1)$ & $19(4.4)$ & $27(7.0)$ & $\begin{array}{l}462 \\
(10.9)\end{array}$ \\
\hline \multicolumn{6}{|c|}{ Residence place, n (\%) } \\
\hline Urban & $2408(90.7)$ & $496(65.9)$ & $355(81.8)$ & $361(94.0)$ & $\begin{array}{l}3620 \\
(85.6)\end{array}$ \\
\hline Rural & $248(9.3)$ & $257(34.1)$ & 79 (18.2) & $23(6.0)$ & $\begin{array}{l}607 \\
(14.4)\end{array}$ \\
\hline \multicolumn{6}{|c|}{ Marital status, n (\%) } \\
\hline Single & $667(25.1)$ & $721(95.8)$ & $263(60.6)$ & $93(24.2)$ & $\begin{array}{l}1744 \\
(41.3)\end{array}$ \\
\hline Married & $1927(72.6)$ & $27(3.6)$ & $165(38.0)$ & $285(74.2)$ & $\begin{array}{l}2404 \\
(56.9)\end{array}$ \\
\hline Others & $62(2.3)$ & $5(0.7)$ & $6(1.4)$ & $6(1.6)$ & $79(1.9)$ \\
\hline \multicolumn{6}{|c|}{ Education level, n (\%) } \\
\hline $\begin{array}{l}\leq \text { High } \\
\text { school }\end{array}$ & $160(6.0)$ & $25(3.3)$ & $53(12.2)$ & $22(5.7)$ & $\begin{array}{l}260 \\
(6.2)\end{array}$ \\
\hline $\begin{array}{l}\text { College and } \\
\text { above }\end{array}$ & $2496(94.0)$ & $728(96.7)$ & $381(87.8)$ & $362(94.3)$ & $\begin{array}{l}3967 \\
(93.8)\end{array}$ \\
\hline
\end{tabular}




\begin{tabular}{|c|c|c|c|c|c|}
\hline Variables & $\begin{array}{l}\text { Medical } \\
\text { workers }(\mathrm{n}= \\
2656)\end{array}$ & $\begin{array}{l}\text { Students(n } \\
=753)\end{array}$ & $\begin{array}{l}\text { General } \\
\text { population }(n= \\
\text { 434) }\end{array}$ & $\begin{array}{l}\text { Public health } \\
\text { professionals(n = } \\
384)\end{array}$ & $\begin{array}{l}\text { Total }(\mathrm{n} \\
=4227)\end{array}$ \\
\hline $\begin{array}{l}\leq 40000 \\
\text { Yuan }\end{array}$ & $431(16.2)$ & $256(34.0)$ & $84(19.4)$ & $43(11.2)$ & $\begin{array}{l}814 \\
(19.3)\end{array}$ \\
\hline $\begin{array}{l}50000- \\
100000 \\
\text { Yuan }\end{array}$ & $1233(46.4)$ & $285(37.8)$ & $185(42.6)$ & $132(34.4)$ & $\begin{array}{l}1835 \\
(43.4)\end{array}$ \\
\hline $\begin{array}{l}110000- \\
350000 \\
\text { Yuan }\end{array}$ & $920(34.6)$ & 178 (23.6) & $137(31.6)$ & $193(50.3)$ & $\begin{array}{l}1428 \\
(33.8)\end{array}$ \\
\hline $\begin{array}{l}>350000 \\
\text { Yuan }\end{array}$ & $72(2.7)$ & $34(4.5)$ & $28(6.5)$ & $16(4.2)$ & $\begin{array}{l}150 \\
(3.5)\end{array}$ \\
\hline \multicolumn{6}{|c|}{ Ever smoking, n (\%) } \\
\hline No & $2369(89.2)$ & $679(90.2)$ & $347(80.0)$ & $339(88.3)$ & $\begin{array}{l}3734 \\
(88.3)\end{array}$ \\
\hline Yes & $287(10.8)$ & $74(9.8)$ & $87(20.0)$ & 45 (11.7) & $\begin{array}{l}493 \\
(11.7)\end{array}$ \\
\hline \multicolumn{6}{|c|}{ Ever drinking, n (\%) } \\
\hline No & $2276(85.7)$ & $668(88.7)$ & $329(75.8)$ & 303 (78.9) & $\begin{array}{l}3576 \\
(84.6)\end{array}$ \\
\hline Yes & $380(14.3)$ & $85(11.3)$ & $105(24.2)$ & $81(21.1)$ & $\begin{array}{l}651 \\
(15.4)\end{array}$ \\
\hline \multicolumn{6}{|c|}{ COVID-19 test results, $n$ (\%) } \\
\hline Positive & $12(0.5)$ & $19(2.5)$ & $6(1.4)$ & $2(0.5)$ & $39(0.9)$ \\
\hline Negative & $2056(77.4)$ & $438(58.2)$ & $272(62.7)$ & $332(86.5)$ & $\begin{array}{l}3098 \\
(73.3)\end{array}$ \\
\hline $\begin{array}{l}\text { Haven't } \\
\text { tested }\end{array}$ & $588(22.1)$ & 296 (39.3) & $156(35.9)$ & $50(13.0)$ & $\begin{array}{l}1090 \\
(25.8)\end{array}$ \\
\hline
\end{tabular}

\subsection{General vaccination behavior and vaccine hesitancy among different population groups}

Differences of general vaccination behavior and vaccine hesitancy in different population groups were shown in Table 2. For public health professionals, $94.3 \%$ of them agreed that vaccines can protect them from diseases, which was higher than in medical workers (90.5\%), students (87.5\%) and general population (88.7\%). Among medical workers, the majority of them $(80.0 \%)$ agreed that they will get all vaccines that the National Immunization Program or government recommended, however, a lower proportion was in students (76.5\%), general population (72.6\%) and public health professionals (75.0\%). When the participants were asked about whether they were hesitated to get vaccination, $39.4 \%$ of general 
population were self-reported "yes" which were higher than in other three population groups. Regarding the sorts of vaccines, they were hesitated or refused, general population had the highest proportion (27.4\%) of those who hesitated to get Pneumococcal Vaccine and the highest proportion of medical workers $(9.8 \%)$ refused to vaccinate against pneumococcal bacteria compared to other groups.

Comparation of the General Vaccine Hesitancy Scale scores in different groups, medical workers had a higher score $(20.33 \pm 8.59)$ than students $(20.32 \pm 8.11)$, general population $(19.87 \pm 7.61)$ and public health professionals $(18.48 \pm 7.51)$. 
Table 2

General vaccination behavior and vaccine hesitancy among participants by different population groups

\begin{tabular}{|c|c|c|c|}
\hline Variables & $\begin{array}{l}\text { Medical } \\
\text { workers }\end{array}$ & Students & $\begin{array}{l}\text { General } \\
\text { population }\end{array}$ \\
\hline
\end{tabular}
$\mathrm{n}(\%)$
n (\%)
n (\%)
$\mathrm{n}(\%)$

Do you agree that vaccines can protect you from diseases?

$\begin{array}{llllll}\text { No } & 253(9.5) & 94(12.5) & 49(11.3) & 22(5.7) & 0.002 \\ \text { Yes } & 2403(90.5) & 659(87.5) & 385(88.7) & 362(94.3) & \end{array}$

Do you agree that you will get all vaccines that the National Immunization Program or government recommended?

$\begin{array}{llllll}\text { No } & 530(20.0) & 177(23.5) & 119(27.4) & 96(25.0) & 0.001 \\ \text { Yes } & 2126(80.0) & 576(76.5) & 315(72.6) & 288(75.0) & \end{array}$

Have you ever hesitated to get vaccination?

$\begin{array}{llllll}\text { No } & 1806(68.0) & 506(67.2) & 263(60.6) & 257(66.9) & 0.026 \\ \text { Yes } & 850(32.0) & 247(32.8) & 171(39.4) & 127(33.1) & \end{array}$

Have you ever refused to get vaccination?

$\begin{array}{llllll}\text { No } & 2222(83.7) & 646(85.8) & 365(84.1) & 320(83.3) & 0.542 \\ \text { Yes } & 434(16.3) & 107(14.2) & 69(15.9) & 64(16.7) & \end{array}$

Have you ever hesitated or refused to get Pneumococcal Vaccine?

$\begin{array}{llllll}\text { No } & 1759(66.2) & 509(67.6) & 293(67.5) & 267(69.5) & 0.000 \\ \begin{array}{l}\text { Ever } \\ \text { hesitated }\end{array} & 636(23.9) & 204(27.1) & 119(27.4) & 82(21.4) & \\ \begin{array}{l}\text { Ever } \\ \text { refused }\end{array} & 261(9.8) & 40(5.3) & 22(5.1) & 35(9.1)\end{array}$

Have you ever hesitated or refused to get Influenza Vaccine?

$\begin{array}{llllll}\text { No } & 1789(67.4) & 514(68.3) & 294(67.7) & 274(71.4) & 0.180 \\ \begin{array}{l}\text { Ever } \\ \text { hesitated }\end{array} & 622(23.4) & 188(25.0) & 109(25.1) & 84(21.9) & 26(6.8) \\ \begin{array}{l}\begin{array}{l}\text { Ever } \\ \text { refused }\end{array} \\ \text { 245 (9.2) }\end{array} & 51(6.8) & 31(7.1) & \end{array}$

The General Vaccine Hesitancy Scale scores

$\begin{array}{llllll}(\text { mean } \pm & 20.33 \pm 8.59 & 20.32 \pm & 19.87 \pm 7.61 & 18.48 \pm 7.51 & 0.001 \\ \text { SD) } & & 8.11 & & \end{array}$




\subsection{COVID-19 vaccine hesitancy and relevant factors among different population groups}

In this study, 64.1\% (2710) of participants were observed having COVID-19 vaccine hesitancy. Among different groups of people, 283 (73.7\%) public health professionals, 1723 (64.9\%) of medical workers, $265(61.1 \%)$ general population and 439 (58.3\%) students were self-reported having COVID-19 vaccine hesitancy. Compared to medical workers, students and public health professionals, the general population had the highest score (10.06 \pm 1.79$)$ of the concerns of COVID-19 epidemic. Regarding to the attitudes of respondents to COVID-19 epidemic, $75.8 \%$ of public health professionals agreed that COVID19 epidemic is a severe problem affecting people's health, which was significantly higher than other groups. And a statistically higher proportion of medical workers (89.9\%) thought that COVID-19 will be a great threat to their health if they infected.

Among different groups, $94.3 \%$ of public health professionals would get COVID-19 vaccine if doctors recommend, more than half of students $(57.6 \%)$ would be affected by their family members or friends, the ratings were significantly higher. Similarly, responses also significantly varied among the four groups $(P<0.01)$ when asked the information about COVID-19 vaccine, public health professionals had the highest proportions of those (90.1\%) who need transparent information about COVID-19 vaccine development, efficacy and safety and $63.5 \%$ had received negative information, $42.0 \%$ of students had doubts about the source of information. When participants were asked if the time costs in waiting for the vaccination or staying at the clinic would be barriers for them to vaccinate, a higher proportion of students were responded "Yes". For public health professionals, $9.6 \%$ of them self-reported that they have no need of getting COVID-19 vaccine because they are healthy, however, $42.2 \%$ of them had gotten emergency COVID-19 vaccination for some reasons (Table 3 ). 
Table 3

Relevant factors of COVID-19 Vaccine Hesitancy among participants by different population groups

\begin{tabular}{llllll} 
Variables & $\begin{array}{l}\text { Medical } \\
\text { workers }\end{array}$ & Students & $\begin{array}{l}\text { General } \\
\text { population }\end{array}$ & $\begin{array}{l}\text { Public health } \\
\text { professionals }\end{array}$ & $P$ \\
\cline { 2 - 4 } & $\mathbf{n}(\%)$ & $\mathbf{n}(\%)$ & $\mathbf{n}(\%)$ & $\mathbf{n}(\%)$ & \\
\hline \multicolumn{2}{l}{ Have COVID-19 vaccine hesitancy } & & \\
\hline No & $933(35.1)$ & $\begin{array}{l}314 \\
(41.7)\end{array}$ & $169(38.9)$ & $101(26.3)$ & \\
\hline Yes & $1723(64.9)$ & $\begin{array}{l}439 \\
(58.3)\end{array}$ & $265(61.1)$ & $283(73.7)$
\end{tabular}

The concern of COVID-19 epidemic

$\begin{array}{lllll}(\text { mean } \pm & 9.83 \pm 2.10 & 9.80 \pm & 10.06 \pm 1.79 & 9.56 \pm 1.88 \\ \text { SD) } & & 2.13 & & \end{array}$

Do you agree that COVID-19 epidemic is a severe problem affecting the health of the community?

$\begin{array}{llllll}\text { No } & 698(26.3) & \begin{array}{l}242 \\ (32.1)\end{array} & 146(33.6) & 93(24.2) & 0.000 \\ \text { Yes } & 1958(73.7) & \begin{array}{l}511 \\ (67.9)\end{array} & 288(66.4) & 291(75.8)\end{array}$

Do you agree that COVID-19 will be a great threat to your health if you are infected?

\begin{tabular}{llllll} 
No & $269(10.1)$ & $\begin{array}{l}108 \\
(14.3)\end{array}$ & $47(10.8)$ & $46(12.0)$ & 0.012 \\
\hline Yes & $2387(89.9)$ & $\begin{array}{l}645 \\
(85.7)\end{array}$ & $387(89.2)$ & $338(88.0)$
\end{tabular}

Do you agree that you will get COVID-19 vaccine if doctors recommend?

\begin{tabular}{llllll} 
No & $221(8.3)$ & $\begin{array}{l}101 \\
(13.4)\end{array}$ & $68(15.7)$ & $22(5.7)$ & 0.000 \\
\hline Yes & $2435(91.7)$ & $\begin{array}{l}652 \\
(86.6)\end{array}$ & $366(84.3)$ & $2435(94.3)$
\end{tabular}

Do you agree that the advice of your family members or friends will affect your intention of getting COVID-19 vaccine?

\begin{tabular}{llllll} 
No & $1721(64.8)$ & $\begin{array}{l}319 \\
(42.4)\end{array}$ & $237(54.6)$ & $254(66.1)$ & 0.000 \\
\hline Yes & $935(35.2)$ & $\begin{array}{l}434 \\
(57.6)\end{array}$ & $197(45.4)$ & $130(33.9)$
\end{tabular}

Do you need transparent information about COVID-19 vaccine development, efficacy and safety?

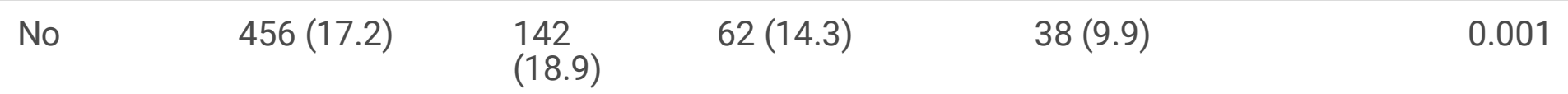




\begin{tabular}{|c|c|c|c|c|c|}
\hline \multirow[t]{2}{*}{ Variables } & $\begin{array}{l}\text { Medical } \\
\text { workers }\end{array}$ & Students & $\begin{array}{l}\text { General } \\
\text { population }\end{array}$ & $\begin{array}{l}\text { Public health } \\
\text { professionals }\end{array}$ & \multirow[t]{2}{*}{$P$} \\
\hline & $\mathrm{n}(\%)$ & $\mathrm{n}(\%)$ & $\mathrm{n}(\%)$ & $\mathrm{n}(\%)$ & \\
\hline Yes & $2200(82.8)$ & $\begin{array}{l}611 \\
(81.1)\end{array}$ & $372(85.7)$ & $346(90.1)$ & \\
\hline \multicolumn{6}{|c|}{ Do you have doubts about the source of information about the COVID-19 vaccine? } \\
\hline No & $2064(77.7)$ & $\begin{array}{l}437 \\
(58.0)\end{array}$ & $276(63.6)$ & $308(80.2)$ & 0.000 \\
\hline Yes & $592(22.3)$ & $\begin{array}{l}316 \\
(42.0)\end{array}$ & $158(36.4)$ & $76(19.8)$ & \\
\hline \multicolumn{6}{|c|}{ Have you ever received negative information about getting the COVID-19 vaccine? } \\
\hline No & $1260(47.4)$ & $\begin{array}{l}373 \\
(49.5)\end{array}$ & $203(46.8)$ & $140(36.5)$ & 0.000 \\
\hline Yes & $1396(52.6)$ & $\begin{array}{l}380 \\
(50.5)\end{array}$ & $231(53.2)$ & $244(63.5)$ & \\
\hline \multicolumn{6}{|c|}{ Would you like to get the COVID-19 vaccine after receiving the negative information about it? } \\
\hline No & $932(35.1)$ & $\begin{array}{l}357 \\
(47.4)\end{array}$ & $205(47.2)$ & $104(27.1)$ & 0.000 \\
\hline Yes & $1724(64.9)$ & $\begin{array}{l}396 \\
(52.6)\end{array}$ & $229(52.8)$ & $280(72.9)$ & \\
\hline \multicolumn{6}{|c|}{$\begin{array}{l}\text { Do you agree that the time costs in waiting for the vaccination or staying at the clinic will be a barrier } \\
\text { for you to get COVID-19 vaccine? }\end{array}$} \\
\hline No & $2033(76.5)$ & $\begin{array}{l}444 \\
(59.0)\end{array}$ & $290(66.8)$ & $281(73.2)$ & 0.000 \\
\hline Yes & $623(23.5)$ & $\begin{array}{l}309 \\
(41.0)\end{array}$ & $144(33.2)$ & $103(26.8)$ & \\
\hline \multicolumn{6}{|c|}{ Do you agree that the environment of the clinic will be a barrier for you to get COVID-19 vaccine? } \\
\hline No & $1708(64.3)$ & $\begin{array}{l}331 \\
(44.0)\end{array}$ & $208(47.9)$ & $217(56.5)$ & 0.000 \\
\hline Yes & $948(35.7)$ & $\begin{array}{l}422 \\
(56.0)\end{array}$ & $226(52.1)$ & $167(43.5)$ & \\
\hline \multicolumn{6}{|c|}{ Do you agree that the costs of going to the clinic will be a barrier for you to get COVID- 19 vaccine? } \\
\hline No & $2011(75.7)$ & $\begin{array}{l}376 \\
(49.9)\end{array}$ & $271(62.4)$ & $270(70.3)$ & 0.000 \\
\hline Yes & $645(24.3)$ & $\begin{array}{l}377 \\
(50.1)\end{array}$ & $163(37.6)$ & $114(29.7)$ & \\
\hline
\end{tabular}




\begin{tabular}{|c|c|c|c|c|c|}
\hline \multirow[t]{2}{*}{ Variables } & $\begin{array}{l}\text { Medical } \\
\text { workers }\end{array}$ & Students & $\begin{array}{l}\text { General } \\
\text { population }\end{array}$ & $\begin{array}{l}\text { Public health } \\
\text { professionals }\end{array}$ & \multirow[t]{2}{*}{$P$} \\
\hline & $\mathrm{n}(\%)$ & $\mathrm{n}(\%)$ & $\mathrm{n}(\%)$ & $\mathrm{n}(\%)$ & \\
\hline No & $2364(89.0)$ & $\begin{array}{l}582 \\
(77.3)\end{array}$ & $354(81.6)$ & $347(90.4)$ & 0.000 \\
\hline Yes & $292(11.0)$ & $\begin{array}{l}171 \\
(22.7)\end{array}$ & $80(18.4)$ & $37(9.6)$ & \\
\hline \multicolumn{6}{|c|}{ Have you gotten emergency COVID-19 vaccination for some reasons? } \\
\hline No & $1610(60.6)$ & $\begin{array}{l}638 \\
(84.7)\end{array}$ & $373(85.9)$ & $222(57.8)$ & 0.000 \\
\hline Yes & $1046(39.4)$ & $\begin{array}{l}115 \\
(15.3)\end{array}$ & $61(14.1)$ & $162(42.2)$ & \\
\hline
\end{tabular}

\subsection{The predictors for COVID-19 vaccine hesitancy among participants}

According with results from the multivariate analyses (Table 4), gender was significantly associated with hesitancy, female was more likely to be hesitant about COVID-19 vaccine than male (OR: $1.247,95 \% \mathrm{Cl}$ : 1.060-1.467). With comparison of general population, public health professionals were more likely to have COVID-19 vaccine hesitancy, while compared to students and medical workers there were no statistically differences. A significant association was noted between household income (past 1 year) and hesitancy $(P<0.001)$, higher levels of household income were significantly associated with higher hesitancy. COVID-19 test results had a significant correlation with vaccine hesitancy, but no significant differences were observed between people who were affected and who were not and also were not reported between those who were affected and who haven't tested. Participants who ever hesitated to get vaccination were more likely to be hesitant (OR: 1.360, 95\% Cl: 1.151-1.608)), but there are no significant differences between those who ever refused to get vaccination and hesitancy. The results showed that respondents' concerns about the epidemic were associated with hesitancy, and those with higher selfreport scores were more likely to be hesitant (OR: 1.068, 95\% Cl: 1.033-1.103). However, a lower score of the General Vaccine Hesitancy Scale was associated with more hesitancy towards COVID-19 vaccination (OR: $0.979,95 \% \mathrm{Cl}: 0.971-0.988$ ). Participants who needed transparent information about COVID-19 vaccine (OR: $1.609,95 \% \mathrm{Cl}$ : 1.343-1.928) and received negative information about the vaccine before (OR: $1.300,95 \% \mathrm{Cl}: 1.130-1.496)$ were both more likely to be hesitant to get COVID-19 vaccine.

\section{Table 4 Analysis of the predictors for COVID-19 vaccine hesitancy among participants}


Variables

Univariate analysis

No Hesitancy

$(n=1517)$
Hesitancy

$(n=2710)$
Multivariate analysis

$P \quad \mathrm{OR}^{*}\left(95 \% \mathrm{Cl}^{*}\right)$
$P$

Age (years), (mean \pm SD)

$\begin{array}{lllll}32.21 \pm 10.02 & 33.47 \pm 9.80 & 0.000 & 1.004(0.994- & 0.424 \\ & & & 1.015)\end{array}$

Gender, n (\%)

Male

572

(37.7)

837 (30.9)

$0.000 \quad 1.000$

Female

945 (62.3)

$1873(69.1)$

$1.247(1.060$ 1.467)

0.008

Ethnicity, n (\%)

Han

$1326(87.4)$

$2439(90.0)$

$0.010 \quad 1.000$

Other

$191(12.6)$

$271(10.0)$

$0.821(0.668-$

1.010)

0.062

Residence place, $\mathrm{n}(\%)$

Urban

1244 (82.0)

2376 (87.7)

$0.000 \quad 1.000$

Rural

2732 (18.0)

334 (12.3)

0.933 (0.763-

0.497

1.140)

Marital status, n (\%)

0.954

Single

692 (45.6)

1052 (38.8)

1607 (59.3)

Married

797 (52.5)

$28(1.8)$

$51(1.9)$

0.939 (0.563-

0.811

Others

Education level, n (\%)

$\leq$ High school

98 (6.5)

$162(6.0)$

$0.531-$

College and above

1419 (93.5)

2548 (94.0)

Population groups

General population

169 (11.1)

265 (9.8)

$0.000 \quad 1.000$

Students

314 (20.7)

439 (16.2)

1.075 (0.829-

1.394)

Medical workers

$933(61.5)$

1723 (63.6)

1.107 (0.880-

1.393)

Public health

101 (6.7)

$283(10.4)$

1.469 (1.069-

0.098 
professionals

2.019)

Household income (past 1 year), n (\%)

0.000

$>350000$

$32(2.1)$

$118(4.4)$

$0.000 \quad 1.000$

$110000-350000$

465 (30.7)

$963(35.5)$

$0.476(0.312-$

$0.726)$

50000-100000

$657(43.3)$

$1178(43.5)$

$0.465(0.306$

$0.707)$

$\leq 40000$

$363(23.9)$

$451(16.6)$

$0.391(0.253-$

$0.605)$

Ever smoking, $\mathbf{n}(\%)$

$\begin{array}{lllll}\text { No } & 1322(87.1) & 2412(89.0) & 0.071 & 1.000 \\ \text { Yes } & 195(12.9) & 298(11.0) & 1.018(0.805- & 0.881\end{array}$

\section{Ever drinking, $\mathrm{n}(\%)$}

No

1256 (82.8)

$2320(85.6)$

$0.015 \quad 1.000$

Yes

261 (17.2)

$390(14.4)$

0.900 (0.731-

0.323

1.109)

COVID-19 test results, $n$ (\%)

0.002

Positive

18 (1.2)

$21(0.8)$

$0.000 \quad 1.000$

Negative

$1024(67.5)$

$2074(76.5)$

$0.996(0.502-$

1.973)

Haven't tested

475 (31.3)

$615(22.7)$

$0.757(0.380-$

1.509)

0.001

0.000

0.000

000

Do you agree that vaccines can protect you from diseases?

$\begin{array}{lllll}\text { No } & 190(12.5) & 228(8.4) & 0.000 & 1.000 \\ \text { Yes } & 1327(87.5) & 2482(91.6) & 1.219(0.978- & 0.078\end{array}$

Do you agree that you will get all vaccines that National Immunization Program or government recommended?

$\begin{array}{llll}\text { No } & 343(22.6) & 579(21.4) & 0.347 \\ \text { Yes } & 1174(77.4) & 2131(78.6) & \end{array}$

Have you ever hesitated to get vaccination?

$\begin{array}{lllll}\text { No } & 1079(71.1) & 1753(64.7) & 0.000 & 1.000 \\ \text { Yes } & 438(28.9) & 957(35.3) & 1.360(1.151- & 0.000\end{array}$


Have you ever refused to get vaccination?

$\begin{array}{lllll}\text { No } & 1309(86.3) & 2244(82.8) & 0.003 & 1.000 \\ \text { Yes } & 208(13.7) & 466(17.2) & & 1.142(0.923- \\ & & & 1.413) & 0.221\end{array}$

Have you ever hesitated or refused to be get Pneumococcal Vaccine?

\begin{tabular}{lllll} 
No & $1032(68.0)$ & $1796(66.3)$ & 0.009 & 1.000 \\
Ever hesitated & $383(25.2)$ & $658(24.3)$ & $0.998(0.810-$ & 0.984 \\
Ever refused & $102(6.7)$ & $256(9.4)$ & $1.229)$ & $1.233(0.892-$ \\
\hline
\end{tabular}

Have you ever hesitated or refused to get Influenza Vaccine?

0.345

No

$1044(68.8)$

$1827(67.4)$

$0.074 \quad 1.000$

Ever hesitated

$366(24.1)$

$637(23.5)$

$0.899(0.730-$

1.107)

Ever refused

$107(7.1)$

$246(9.1)$

$1.116(0.814-$ 1.532)

The General Vaccine Hesitancy Scale scores

(mean $\pm S D)$

$$
21.01 \pm 8.07
$$

$19.61 \pm 8.43$

0.000

$0.979(0.971-$

$0.988)$

0.316

0.495

The concern of COVID-19 epidemic
(mean \pm SD)
$9.65 \pm 2.00$
$9.92 \pm 2.08$
0.000
$1.068(1.033-$
0.000
$1.103)$

Do you agree that COVID-19 epidemic is a severe problem affecting the health of the community?

$\begin{array}{lllll}\text { No } & 470(31.0) & 709(26.2) & 0.001 & 1.000 \\ \text { Yes } & 1047(69.0) & 2001(73.8) & 1.080(0.926- & 0.327\end{array}$

Do you agree that COVID-19 will be a great threat to your health if you are infected?

$\begin{array}{lllll}\text { No } & 205(13.5) & 265(9.8) & 0.000 & 1.000 \\ \text { Yes } & 1312(86.5) & 2445(90.2) & 1.100(0.881- & 0.401\end{array}$

Do you agree that you will get COVID-19 vaccine if doctors recommend?

$\begin{array}{lllll}\text { No } & 170(11.2) & 242(8.9) & 0.017 & 1.000 \\ \text { Yes } & 1347(88.8) & 2468(91.1) & 0.873(0.687- & 0.267\end{array}$


Do you agree that the advice of your family members or friends will affect your intention of getting COVID-19 vaccine?

$\begin{array}{llll}\text { No } & 909(59.9) & 1622(59.9) & 0.965- \\ \text { Yes } & 608(40.1) & 1088(40.1)\end{array}$

Do you agree that you have no need of getting COVID-19 vaccine because you are healthy?

\begin{tabular}{|c|c|c|c|c|c|}
\hline No & $1280(84.4)$ & 2367 (87.3) & 0.007 & 1.000 & \\
\hline Yes & $237(15.6)$ & $343(12.7)$ & & $\begin{array}{l}0.827(0.678- \\
1.009)\end{array}$ & 0.062 \\
\hline
\end{tabular}

Do you need transparent information about COVID-19 vaccine development, efficacy and safety?

$\begin{array}{lllll}\text { No } & 347(22.9) & 351(13.0) & 0.000 & 1.000 \\ \text { Yes } & 1170(77.1) & 2359(87.0) & 1.609(1.343- & 0.000\end{array}$

Do you have doubts about the source of information about the COVID-19 vaccine?

$\begin{array}{llll}\text { No } & 1129(74.4) & 1956(72.2) & 0.115- \\ \text { Yes } & 388(25.6) & 754(27.8)\end{array}$

Have you ever received negative information about getting the COVID-19 vaccine?

$\begin{array}{lllll}\text { No } & 817(53.9) & 1159(42.8) & 0.000 & 1.000 \\ \text { Yes } & 700(46.1) & 1551(57.2) & 1.300(1.130- & 0.000\end{array}$

Would you like to get the COVID-19 vaccine after receiving the negative information about it?

$\begin{array}{lllll}\text { No } & 652(43.0) & 946(34.9) & 0.000 & 1.000 \\ \text { Yes } & 865(57.0) & 1764(65.1) & 1.143(0.985- & 0.079 \\ & & & 1.325) & \end{array}$

Do you agree that the time costs in waiting for the vaccination or staying at the clinic will be a barrier for you to get COVID-19 vaccine?

$\begin{array}{llll}\text { No } & 1087(71.7) & 1961(72.4) & 0.623 \\ \text { Yes } & 430(28.3) & 749(27.6)\end{array}$

Do you agree that the environment of the clinic will be a barrier for you to get COVID-19 vaccine?

$\begin{array}{llll}\text { No } & 892(58.8) & 1572(58.0) & 0.616- \\ \text { Yes } & 625(41.2) & 1138(42.0)\end{array}$

Do you agree that the cost of going to the clinic will be a barrier for you to get COVID-19 vaccine?

No

1030 (67.9)

1898 (70.0)

$0.148-$ 
Have you gotten emergency COVID-19 vaccination for some reasons?

$\begin{array}{lllll}\text { No } & 1056(69.6) & 1787(65.9) & 0.015 & 1.000 \\ \text { Yes } & 461(30.4) & 923(34.1) & 1.030(0.886- & 0.704\end{array}$

*Abbreviations: OR, odds ratio; Cl: confidence interval.

\section{Discussion}

In current study, $64.1 \%$ of all participants were self-reported having COVID-19 vaccine hesitancy. While $35.9 \%$ were non-hesitant for get vaccination against COVID-19, this rate was lower than the findings in most of the previous other studies, $77.6 \%$ in France, $91.3 \%$ in China, $74.5 \%$ in India, $70.8 \%$ in Italy and $62.6 \%$ in Ethiopia (21-24). Among the study participants, the public health professionals $(73.7 \%)$ had the highest rate of hesitancy, followed by medical workers (64.9\%), general population (61.0\%) and students (58.4\%). Compared to the hesitancy rate of $47.5 \%$ among college students in United states, it was noteworthy that the Chinese college students had a higher rate of hesitancy toward COVID-19 vaccine (9, $25,26)$. Apart from the change of people's attitude with the increasingly severe COVID-19 pandemic over the world and different study time, different socio-demographic factors, levels of health literacy, particularly with respect to immunization schedules, as well as changes in local health policies over time and advances in the development of the COVID-19 vaccines are possible reasons of the differences on COVID-19 vaccine hesitancy. At present, the COVID-19 vaccine is available in China, however, not everyone in the cities or areas with accessible vaccination has been vaccinated. This level of hesitancy is very likely to be a barrier to or delay the establishment of herd immunity.

Among different population groups, this study indicated that public health professionals were more likely to have COVID-19 vaccine hesitancy than the general population. Most of the public health professionals in our study were healthcare workers from local CDCs who had more specialized knowledge. Previous studies had reported that healthcare workers or providers were instead more likely to have a low willingness to be vaccinated $(27,28)$. Public health professionals did not want to receive the shot might probably due to their low perception of risk on contracting the disease, or they would pay more attention to individual protection. On the other hands, they might consider the COVID-19 as common flu and not a much dangerous disease, or thought that they would be infected even though they get vaccination, due to the doubts of the efficacy of the vaccine. Additionally, we assumed public health professionals had more sources or approaches to receive information about the vaccine, including some negative news or information, which in turn affected their willingness to get a shot to some extent(29). Public health professionals always serve as the most trusted advisors and influencers of vaccination decisions and their recommendations are important facilitators for vaccine acceptance (28). If the attitudes or behaviors of them towards getting COVID-19 vaccine are hesitant, the public would have doubts or concerns about the vaccine. Therefore, interventions focusing on increasing the health care workers' 
willingness to be vaccinated is an urgent need. Another study showed that knowledge about particular vaccines, their efficacy and safety, would help healthcare workers to build their own confidence in vaccines and willingness to recommend vaccines to others (30). To develop education campaigns based on accurate and authoritative knowledge or information might be working for reducing their hesitancy.

Besides, participants who have ever hesitated to get vaccination were more likely to be hesitant for the vaccination against COVID-19. Similarly, another survey found that a prominent determinant of COVID-19 vaccine avoidance is whether a person had the flu shot in the last two years (31). In our research, a lower score of the General Vaccine Hesitancy Scale was correlated to a higher possibility to be hesitant towards COVID-19 vaccines. It is surprising those who were not hesitant against other vaccines during pre-COVID era were more likely to be hesitant towards the COVID-19 vaccine. This might because the COVID-19 epidemic has posed unprecedented health threats to the public's health and the COVID-19 vaccines were not undergone long-term clinical trials as others for emergency use as a strategy of controlling the pandemic. All individuals may have preoccupation and anxiety on safety and efficacy issues of COVID-19 vaccination, even if they were not previously hesitant to uptake the general vaccines. Therefore, the concerns about vaccine's safety and efficacy is one of the most important sources of vaccine hesitancy, as demonstrated in as other studies (32).

In the past several months, the negative news about the safety and efficacy of COVID-19 vaccines have been widely spread on the Internet, both before and during vaccination. During the COVID-19 pandemic, governments and the public were unavoidably exposed to huge amount of rapid and far-reaching spread of questionable information, namely infodemic. Fake news and misinformation have become prevalent in various social media and have been skyrocketed since the beginning of the COVID-19 pandemic(33). Some media or websites would exaggerate or make unreliable news, because negative tweets expressing misinformation on vaccine confidence and even rumors, had more followers and attracted more public engagement online (34). Our study highlighted the needs of transparent information about COVID-19 vaccine development, efficacy and safety and the negative roles of misinformation on COVID-19 vaccine in vaccine hesitancy. The COVID-19 vaccine was developed at an alarming rate, unlike other vaccines in the past, so people may have more concerns about it, which has frequently been reported as the major obstacle to vaccination decision-making, especially for newly introduced vaccines which have not been fully tested in the real world $(21,35,36)$. Meanwhile, risk information in the news had direct effects on people's health behavior during the pandemic (37). Indeed, some other studies reported perceived effectiveness of the vaccine increased vaccination intention $(32,38)$. Therefore, providing reliable information on the disease and the relevant vaccines by the authorities and establishing suitable communication strategies between the government and the public would be helpful to create a trustworthy environment and hence improve public's confidence in vaccination. Also, a warning system on infodemic is needed, apart from the monitoring of the disease itself in the similar circumstances.

In the information age, people can be easily influenced by false information and negative news, and thus may led to fear or panic and make arbitrary decisions (39). In the meantime, social medias play an increasingly important role in spreading both accurate information and misinformation, hence we can

Page $21 / 26$ 
also use medias or websites to inform people in a positive way. To increase vaccination rates, information and education campaigns on the safety and efficacy of the COVID-19 vaccine will enhance public confidence in the COVID-19 vaccine $(40,41)$. People who have received vaccines, such as respected celebrities, those with influencing popularity and health care professionals with high click rate can be used as vaccination advocates to promote the benefits of vaccines to increase public's willingness of vaccination (42).

In general, our study suggested a high prevalence of vaccine hesitancy against the COVID-19 vaccine among Chinese, especially among public health professionals. In addition, the spread of inaccurate information can cause, even increase vaccine hesitancy. Nowadays, especially after the outbreak, people are more likely to get information about vaccines on the Internet, which always containing incorrect, false and negative information. As a result, people may have further doubts about the safety or efficacy of the vaccine and refusal or delay vaccination. Consequently, proactive work against possible misinformation is needed during the development and vaccination phases of the COVID-19 vaccine, and more focused and nuanced public health information from trusted members of the population, including physicians, journalists, and experts is necessary and useful. More importantly, educating social media users on how to distinguish reliable information and to encourage them not to circulate false information are helpful for tackling COVID-19-related misinformation (39). For healthcare workers, to improve their knowledge, confidence of COVID-19 vaccine and communication skills is essential both for their own health and for the guiding effects on the general public. However, vaccine hesitancy is a complex phenomenon that does not depend only on a sufficient knowledge of vaccinations or perceived correct information, further study is necessary to explore other factors and effective prevention or intervention to reduce vaccine hesitancy.

\section{Limitations}

Our survey was conducted before the COVID-19 vaccine became widely available in China. Therefore, there might have differences between the prevalence of hesitancy in our study and the actual vaccination rate later on. However, our study still provided meaningful results in terms of the prevalence and related factors of the vaccine hesitancy among various populations. Although four different population groups were surveyed in our study, the generalizability of our results will still be limited in certain aspects due to the nonprobability sampling we used. Also, the questionnaires were self-reported online, thus the information bias probably existed in this study.

\section{Conclusions}

Given the influence of public health workers on vaccination acceptance of public and the relatively high rate of vaccine hesitancy among them, providing appropriate information to them and training on communicating skills are of most important for their own benefit and the public's. Since the negative information of COVID-19 vaccine could cause and increase vaccine hesitancy, transparent information about the vaccine was vital for public to make vaccination decisions. Evidence-based information 
strategies where the potential benefits and risks of vaccination are properly explained, reducing the spread of misinformation and disseminating facts in a timely and accurate way, will likely reduce vaccine hesitancy.

\section{Declarations}

\section{Ethics approval and consent to participate}

This study has been approved by the Ethical Review Committee of Chinese Center for Disease Control and Prevention on December 4th, 2020 (approval number: 202020). An electronic informed consent was provided before the start of the questionnaire survey, upon completion of the informed consent, participants filled in the on-line questionnaire.

\section{Consent for publication}

Not applicable.

\section{Availability of data and materials}

The datasets used and/or analysed during the current study are available from the corresponding author on reasonable request.

\section{Competing interests}

The authors declare that they have no competing interests.

\section{Funding}

This study was funded by the Asian Regional Special Cooperation Fund of National Health Commission of the People's Republic of China (BLXM01).

\section{Authors' contributions}

Xiaoyou Su, Yiman Huang prepared the first draft. Xiaoyou Su, Youlin Qiao and Yiman Huang managed the overall project. Mingyu Si, Weijun Xiao and Hao Wang provided writing-review. Xiaoyou Su, Wenjun Wang, Xiaofen Gu, Li Ma, Li Li, Shaokai Zhang, Chunxia Yang and Yanqin Yu were responsible for the questionnaire survey of people in seven geographical regions of China. Xiaoyou Su and Yiman Huang analyzed data.

\section{Acknowledgements}

The authors acknowledge the contributions of all associated workers and students of the seven collaborating institutions for the support in data collection.

\section{Authors' information}


${ }^{1}$ School of Population Medicine and Public Health, Chinese Academy of Medical Sciences and Peking Union Medical College, Beijing, China; ${ }^{2}$ School of Nursing, Jining Medical University, Jining, Shandong, China; ${ }^{3}$ Affiliated Tumor Hospital, Xinjiang Medical University, Urumqi, China; ${ }^{4}$ Public Health School, Dalian Medical University, Dalian, China; ${ }^{5}$ Department of Clinical Research, The First Affiliated Hospital, Jinan University, Guangzhou, China; ${ }^{6}$ Henan Cancer Hospital, Affiliate Cancer Hospital of Zhengzhou University, Zhengzhou, China; ${ }^{7}$ Department of Epidemiology and Biostatistics, West China School of Public Health and West China Fourth Hospital, Sichuan University, Chengdu, Sichuan, China; ${ }^{8}$ School of Public Health, Department of Clinical Research, Baotou Medical College, Baotou, China; ${ }^{9}$ Department of Cancer Epidemiology, National Cancer Center/National Clinical Research Center for Cancer/Cancer Hospital, Chinese Academy of Medical Sciences and Peking Union Medical College, 17 South Panjiayuan, Chaoyang District, Beijing, China.

\section{References}

1. Romano CM, Chebabo A, Levi JE. Past, present, and future of COVID-19: a review. Braz J Med Biol Res. 2020;53(9):e10475.

2. Yang P, Wang X. COVID-19: a new challenge for human beings. Cellular \& molecular immunology. 2020;17(5):555-7.

3. Ansari-Moghaddam A, Seraji M, Sharafi Z, Mohammadi M, Okati-Aliabad H. The protection motivation theory for predict intention of COVID-19 vaccination in Iran: a structural equation modeling approach. BMC Public Health. 2021;21(1):1165.

4. Dube E, Laberge $C$, Guay M, Bramadat P, Roy R, Bettinger J. Vaccine hesitancy: an overview. Hum Vaccin Immunother. 2013;9(8):1763-73.

5. Kestenbaum LA, Feemster KA. Identifying and addressing vaccine hesitancy. Pediatr Ann. 2015;44(4):e71-5.

6. MacDonald NE, Hesitancy SWGoV. Vaccine hesitancy: Definition, scope and determinants. Vaccine. 2015;33(34):4161-4.

7. Dubé E, MacDonald NE. How can a global pandemic affect vaccine hesitancy? Expert review of vaccines. 2020;19(10):899-901.

8. Lazarus JV, Ratzan SC, Palayew A, Gostin LO, Larson HJ, Rabin K, et al. A global survey of potential acceptance of a COVID-19 vaccine. Nat Med. 2020.

9. Sun Y, Chen X, Cao M, Xiang T, Zhang J, Wang P, et al. Will Healthcare Workers Accept a COVID-19 Vaccine When It Becomes Available? A Cross-Sectional Study in China. Frontiers in public health. 2021;9:664905.

10. Pogue K, Jensen JL, Stancil CK, Ferguson DG, Hughes SJ, Mello EJ, et al. Influences on Attitudes Regarding Potential COVID-19 Vaccination in the United States. Vaccines (Basel). 2020;8(4).

11. Reiter PL, Pennell ML, Katz ML. Acceptability of a COVID-19 vaccine among adults in the United States: How many people would get vaccinated? Vaccine. 2020;38(42):6500-7. 
12. Malik AA, McFadden SM, Elharake J, Omer SB. Determinants of COVID-19 vaccine acceptance in the US. EClinicalMedicine. 2020;26:100495.

13. Salali GD, Uysal MS. COVID-19 vaccine hesitancy is associated with beliefs on the origin of the novel coronavirus in the UK and Turkey. Psychological medicine. 2020:1-3.

14. Launay O. Risks of non-vaccination. Med Mal Infect. 2020;50(1):1-2.

15. Liau A, Zimet GD, Fortenberry JD. Attitudes about human immunodeficiency virus immunization: the influence of health beliefs and vaccine characteristics. Sexually transmitted diseases. 1998;25(2):7681.

16. Head KJ, Kasting ML, Sturm LA, Hartsock JA, Zimet GD. A National Survey Assessing SARS-CoV-2 Vaccination Intentions: Implications for Future Public Health Communication Efforts. Science Communication. 2020.

17. Larson HJ, Jarrett C, Schulz WS, Chaudhuri M, Zhou Y, Dube E, et al. Measuring vaccine hesitancy: The development of a survey tool. Vaccine. 2015;33(34):4165-75.

18. Shapiro GK, Tatar O, Dube E, Amsel R, Knauper B, Naz A, et al. The vaccine hesitancy scale: Psychometric properties and validation. Vaccine. 2018;36(5):660-7.

19. Opel DJ, Mangione-Smith R, Taylor JA, Korfiatis C, Wiese C, Catz S, et al. Development of a survey to identify vaccine-hesitant parents: the parent attitudes about childhood vaccines survey. Hum Vaccin. 2011;7(4):419-25.

20. Opel DJ, Taylor JA, Mangione-Smith R, Solomon C, Zhao C, Catz S, et al. Validity and reliability of a survey to identify vaccine-hesitant parents. Vaccine. 2011;29(38):6598-605.

21. Wang J, Jing R, Lai X, Zhang H, Lyu Y, Knoll MD, et al. Acceptance of COVID-19 Vaccination during the COVID-19 Pandemic in China. Vaccines (Basel). 2020;8(3).

22. Detoc M, Bruel S, Frappe P, Tardy B, Botelho-Nevers E, Gagneux-Brunon A. Intention to participate in a COVID-19 vaccine clinical trial and to get vaccinated against COVID-19 in France during the pandemic. Vaccine. 2020;38(45):7002-6.

23. Abebe H, Shitu S, Mose A. Understanding of COVID-19 Vaccine Knowledge, Attitude, Acceptance, and Determinates of COVID-19 Vaccine Acceptance Among Adult Population in Ethiopia. Infection and drug resistance. 2021;14:2015-25.

24. Lazarus JV, Ratzan SC, Palayew A, Gostin LO, Larson HJ, Rabin K, et al. A global survey of potential acceptance of a COVID-19 vaccine. Nat Med. 2021;27(2):225-8.

25. Sharma M, Davis RE, Wilkerson AH. COVID-19 Vaccine Acceptance among College Students: A Theory-Based Analysis. Int J Environ Res Public Health. 2021;18(9).

26. Bai W, Cai H, Liu S, Liu H, Qi H, Chen X, et al. Attitudes toward COVID-19 vaccines in Chinese college students. International journal of biological sciences. 2021;17(6):1469-75.

27. Paoli S, Lorini C, Puggelli F, Sala A, Grazzini M, Paolini D, et al. Assessing Vaccine Hesitancy among Healthcare Workers: A Cross-Sectional Study at an Italian Paediatric Hospital and the Development of a Healthcare Worker's Vaccination Compliance Index. Vaccines (Basel). 2019;7(4). 
28. Lin C, Tu P, Beitsch LM. Confidence and Receptivity for COVID-19 Vaccines: A Rapid Systematic Review. Vaccines (Basel). 2020;9(1).

29. CastañedaasVasquez DE, Ruiz-Padilla JP, Botello-Hernandez E. Vaccine Hesitancy against SARSCoV-2 in Health Personnel of Northeastern Mexico and its Determinants. Journal of occupational and environmental medicine. 2021.

30. Paterson P, Meurice F, Stanberry LR, Glismann S, Rosenthal SL, Larson HJ. Vaccine hesitancy and healthcare providers. Vaccine. 2016;34(52):6700-6.

31. Thunström L, Ashworth M, Finnoff D, Newbold SC. Hesitancy Toward a COVID-19 Vaccine. EcoHealth. 2021:1-17.

32. Edwards B, Biddle N, Gray M, Sollis K. COVID-19 vaccine hesitancy and resistance: Correlates in a nationally representative longitudinal survey of the Australian population. PLoS One. 2021;16(3):e0248892.

33. The Lancet Infectious D. The COVID-19 infodemic. The Lancet Infectious diseases. 2020;20(8):875.

34. Hou Z, Tong Y, Du F, Lu L, Zhao S, Yu K, et al. Assessing COVID-19 vaccine hesitancy, confidence and public engagement: a global social listening study. Journal of medical Internet research. 2021.

35. Yaqub O, Castle-Clarke S, Sevdalis N, Chataway J. Attitudes to vaccination: a critical review. Social science \& medicine (1982). 2014;112:1-11.

36. Dubé E, Macdonald NE. Chapter 26 - Vaccine Acceptance: Barriers, Perceived Risks, Benefits, and Irrational Beliefs: The Vaccine Book; 2016.

37. Bursztyn L, Rao A, Roth C, Yanagizawa-Drott DJTWERPS. Misinformation during a Pandemic. 2020.

38. Dong D, Xu RH, Wong EL, Hung CT, Feng D, Feng Z, et al. Public preference for COVID-19 vaccines in China: A discrete choice experiment. Health expectations : an international journal of public participation in health care and health policy. 2020;23(6):1543-78.

39. Gabarron E, Oyeyemi SO, Wynn R. COVID-19-related misinformation on social media: a systematic review. Bulletin of the World Health Organization. 2021;99(6):455-63a.

40. Jiang S. Don't rush to deploy COVID-19 vaccines and drugs without sufficient safety guarantees. Nature. 2020;579(7799):321.

41. Miyachi T, Takita M, Senoo Y, Yamamoto K. Lower trust in national government links to no history of vaccination. Lancet (London, England). 2020;395(10217):31-2.

42. Al-Metwali BZ, Al-Jumaili AA, Al-Alag ZA, Sorofman B. Exploring the acceptance of COVID-19 vaccine among healthcare workers and general population using health belief model. Journal of evaluation in clinical practice. 2021. 